\title{
Cuidado(!) na prevenção do câncer: ética, danos e equívocos
}

\author{
Caution(!) in cancer prevention: ethics, damages and misconceptions \\ ¡Cuidado(!) en la prevención del cáncer: ética, daños y equívocos
}

Charles Dalcanale Tesser. Universidade Federal de Santa Catarina (UFSC). Florianópolis, SC, Brasil. charlestesser@ccs.ufsc.br (Autor correspondente)

O artigo Principios Éticos y Prevención Cuaternaria: ¿es posible no proteger el ejercicio del principio de autonomía? é preciso em criticar a medicalização social que tem avançado na direção da prevenção. A prevenção é uma ideia apelativa, popular e medicamente defensável. O envelhecimento populacional, o sedentarismo progressivo, a epidemia de obesidade e a proliferaçáo de doenças crônicas, bem como as limitaçôes terapêuticas da biomedicina do século XX e XXI, reforçam essa ideia. Quase todos preferem ou apoiam o dito popular "antes prevenir que remediar".

O problema é que tal senso comum é perigoso e por vezes deve ser restrito, evitado ou mesmo combatido e reorientado, particularmente quando estão envolvidas intervençóes (diagnósticas e terapêuticas) com alto potencial de dano, especialmente quando se chega ao extremo de impor procedimentos preventivos em situaçóes em que o fundamento é precário, como na prevenção do câncer por meio da mamografia periódica no Uruguai. Além disso, as pessoas podem não querer realizar os procedimentos preventivos, pelo que não devem ser penalizadas de modo algum. Mesmo diante da mais consensual evidência, o que cabe ao profissional e ao Sistema de Saúde é aconselhar e informar; não deve haver imposição de condutas preventivas, especialmente se não houver consequências para a coletividade, como é o caso. Alguns pontos merecem destaque para compreensão da situação.

Primeiro: na prevenção, as implicaçôes éticas são - e devem ser - diferentes em relação às intervençôes em pessoas adoecidas clinicamente. Na prevenção, exige-se maior rigor quanto à garantia de benefícios e evitação de danos, pois a intervenção ocorrerá em pessoas de baixo risco (assintomáticas) em que deve imperar mais fortemente o primum non nocere. Essa garantia e segurança exigem sólidos critérios científicos que somente podem ser obtidos por meio de evidências atualizadas, resultantes de metaanálises e de ensaios clínicos de alta qualidade denominados como ensaios clínicos controlados e aleatorizados. Portanto, a opiniáo pessoal ou coletiva de especialistas - quaisquer que sejam, médicos de família ou especialistas focais - não deve vigorar quanto aos rastreamentos. Contudo, é compreensível que mastologistas e ginecologistas tenham opiniōes favoráveis ao rastreamento do câncer de mama mais cedo, porque eles entendem e tratam os "cânceres de mama clinicamente relevantes" e os "cânceres produzidos pelo rastreamento" como fenômenos idênticos, dada a impossibilidade técnica atual em diferenciar "os cânceres clinicamente relevantes" dos sobrediagnósticos resultantes do rastreamento. Tal pressão situacional e emocional, porém, não deve definir recomendaçóes de rastreamento. Ao contrário, essa opinião deve ser informada e continuamente reformulada por evidências atualizadas de boa qualidade, que estão além da experiência pessoal e são contraintuitivas, mas hoje acessíveis facilmente.

\section{Palavras-chave:}

Programas de Rastreamento Mamografia

Medicalização

Neoplasias da Mama

Prevenção Quaternária

\section{Keywords:}

Mass Screening Mammography Medicalization Breast Neoplasms Quaternary Prevention

\section{Palabras clave:} Tamizaje Masivo Mamografía Medicalización Neoplasias de la Mama Prevención Cuaternaria 
No caso da mamografia, os danos conhecidos envolvem exposição à radiação ionizante ao longo de vários anos; mastectomias desnecessárias devido ao sobrediagnóstico e sobretratamento; sofrimento psicológico quanto aos falsos positivos (com consequentes biópsias desnecessárias e complicações associadas) e falsos negativos; situaçóes borderlines que necessitam de seguimento por vários anos, convertendo sadios em doentes; maior mortalidade devido a insuficiência cardíaca, bem como induçáo de câncer de pulmão. ${ }^{1}$ Nos rastreamentos, o ônus da prova fica com a "intervençáa": deve haver provas (evidências) de que os benefícios superam amplamente os danos. Em caso de dúvida, estáo indicadas, pelo princípio da precaução e do primum non nocere, prudência e conduta conservadora: não intervir e discutir o balanço danos versus benefícios com a pessoa, à luz da informaçáo disponível.

Segundo: a redução da mortalidade específica é o principal, se não quase o único, critério para avaliaçáo dos benefícios de rastreamentos de câncer. Sua estimativa em meta-análises recentes e rigorosas de ensaios clínicos envolvendo rastreamentos por mamografia reduziu-se para $15 \%,{ }^{2}$ o que é pouco ou nada divulgado para o público. Além disso, nas séries históricas de mortalidade por câncer de mama nas populações, tal redução não é observada, e espera-se algum correlato nos indicadores populacionais dos resultados dos ensaios clínicos. O mais longo estudo histórico a respeito não mostra diferença significativa na mortalidade por câncer de mama entre populaçôes rastreadas e não rastreadas. ${ }^{3}$ Nele, a redução pequena de mortalidade em ambas as populaçôes é atribuída à melhoria da terapêutica. Esse tipo de evidência é importante. Por exemplo, ela tem sustentado a indicação do rastreamento de câncer cervicouterino há muitos anos, cuja redução histórica da mortalidade é grande e tem sido atribuída à introdução do rastreamento a partir de 1950-1960. ${ }^{4}$

Terceiro: os danos do rastreamento por mamografia existem em grande proporção. Talvez os mais importantes sejam os falsos positivos e principalmente o sobrediagnóstico, situação em que o "problema" diagnosticado pelo rastreamento não teria consequências clínicas na vida da pessoa. ${ }^{5,6}$ Devido à incapacidade atual de distinção entre os "verdadeiros" casos curados precocemente, que se manifestariam clinicamente, daqueles que náo se manifestariam (embora o "diagnóstico" tenha sido realizado corretamente), o sobrediagnóstico gera o sobretratamento - todos são igualmente tratados - e também o chamado "paradoxo da popularidade": todas as mulheres sobrediagnosticadas e sobretratadas (prejudicadas) pelo rastreamento consideram-se as (bem poucas) beneficiadas por ele, reforçando o senso comum anteriormente mencionado. Por isso, há proposta de mudança de nomenclatura para os diagnósticos produzidos por rastreamento, que mereceriam distinção dos cânceres diagnosticados clinicamente. ${ }^{7}$ A dimensão do sobrediagnóstico variou nos estudos de $10 \%{ }^{8}$ até $52 \%{ }^{9}$ das pessoas diagnosticadas por mamografia periódica. Recentes estudos de boa qualidade concluíram que essa proporção é de $30 \%{ }^{1}$ De cada 2.000 mulheres rastreadas ao longo de 10 anos, uma mulher terá sua vida salva pelo rastreamento; 10 serão sobrediagnosticadas e sobretratadas; e 200 mulheres receberão um resultado falso positivo, levando a estresses psicológicos e a biópsias desnecessárias. ${ }^{1,10}$

Quarto: para agravar o quadro, a investigação dos danos dos rastreamentos tem se mostrado aquém do que se imagina. Os ensaios clínicos raramente se dispóem a quantificar os danos. ${ }^{11}$ Apenas $13 \%$ dos artigos sobre o rastreamento de câncer de mama citam o sobrediagnóstico como uma possibilidade, havendo ainda uma grande diferença entre periódicos científicos de medicina geral e de especialidades focais: estas últimas são mais explícitas em rejeitar dados de revisões sistemáticas e apresentam com maior frequência problemas de conflitos de interesses. ${ }^{12}$

Concluindo, os critérios éticos e técnicos envolvidos e os estudos dos últimos anos questionam a recomendação do rastreamento por mamografia em qualquer idade, o que já seria motivo para revisão das recomendaçóes ou, no mínimo, sua relativização e abertura de discussão do problema com as mulheres e a sociedade. Logo, a obrigatoriedade da realização do rastreamento por mamografia é um desserviço à saúde das mulheres do Uruguai. No Brasil, pelos mesmos pontos anteriormente abordados, merece crítica enfática a campanha "Outubro Rosa", em que instituições privadas e filantrópicas especializadas em câncer de mama (espantosamente) recomendam o rastreamento a partir dos 40 anos ${ }^{13-15}$ e o governo brasileiro a partir dos 50 anos, ${ }^{16} \mathrm{sem}$ abertura para compartilhamento de decisão com as mulheres devidamente informadas, nem informaçóes claras sobre os danos. Cabe revisão, pelas autoridades sanitárias brasileiras, da indicação da mamografia a partir dos 50 anos ou dos termos dessa recomendação, abrindo discussão sobre o tema. Também merece severa - ou ainda maior crítica - a equivalente campanha preventiva "Novembro Azul", assumida pelo governo brasileiro, que orienta explicitamente a população masculina assintomática a demandar exames preventivos de câncer de próstata, sem base de evidência para tal. Site oficial do governo federal é explícito: "Homens a partir dos 50 anos devem procurar um posto de saúde para realizar exames de rotina", ${ }^{17}$ "A Sociedade Brasileira de Urologia recomenda que todos os homens com 45 anos de idade ou mais façam um exame de próstata anualmente, o que compreende o toque retal feito e o PSA", ${ }^{17}$ apesar de o próprio Ministério da Saúde brasileiro não recomendar açôes de rastreamento de câncer de próstata ${ }^{16,18}$ e, inclusive, haver 
consensual recomendação contrária derivada de avaliação recente de evidências por instituiçôes idôneas quanto ao rastreamento com PSA (Prostate-Specific Antigen). ${ }^{19}$ Tudo isso é um verdadeiro desafio individual e coletivo de prevenção quaternária, ${ }^{20,21}$ e esperamos que as autoridades sanitárias brasileiras e uruguaias e os médicos de família e comunidade, a exemplo do Dr. Pizzanelli, se mobilizem para corrigir esses equívocos e socializar informações e orientaçóes bem fundamentadas a esse respeito, bem como orientar, discutir e compartilhar decisóes com seus usuários.

\section{Referências}

1. Gøtzsche PC, Jørgensen KJ. Screening for breast cancer with mammography. The Nordic Cochrane Centre; 2012. Cochrane Database Syst Rev. 2013 Jun 4;6:CD001877. http://dx.doi.org/10.1002/14651858.CD001877.pub5

2. Welch HG, Frankel BA. Likelihood that a woman with screen-detected breast cancer has had her "life saved" by that screening. Arch Intern Med. 2011 Dec;171(22):2043-6. PMid:22025097. http://dx.doi.org/10.1001/archinternmed.2011.476

3. Jørgensen KJ, Zahl Per-Henrik, Gøtzsche P. Breast cancer mortality in organised mammography screening in Denmark: comparative study. BMJ. 2010;340:c1241. PMid:20332505 PMCid:PMC2844939. http://dx.doi.org/10.1136/bmj.c1241

4. Boyle P, Levin B; World Health Organization - WHO, International Agency for Research on Cancer. World Cancer Report 2008. Lyon, 2008. Disponível em: http://www.iarc.fr/en/publications/pdfs-online/wcr/2008/.

5. Black WC, Welch HG. Overdiagnosis in cancer. JNCl. 2010;102(9)605-613. http://dx.doi.org/10.1093/jnci/djq099

6. Welch HG. Overdiagnosed: Making people sick in the pursuit of health. Boston: Beacon Press; 2011.

7. Esserman LJ, Reid B, Thompson IM. Overdiagnosis and overtreatment in cancer: An opportunity for improvement. JAMA. 2013;310(8):797-798. PMid:23896967. http://dx.doi.org/10.1001/jama.2013.108415

8. Kopans DB, Smith RA, Duffy SW. Mammographic screening and "overdiagnosis". Radiology. 2011;260(3):616-20. PMid:21846757. http://dx.doi.org/10.1148/radiol.11110716

9. Jørgensen KJ, Gøtzsche PC. Overdiagnosis in publicly organised mammography screening programmes: systematic review of incidence trends. BMJ. 2009;339:b2587. http://dx.doi.org/10.1136/bmj.b2587

10. Bleyer A, Welch G. Effect of Three Decades of Screening Mammography on Breast-Cancer Incidence. N Engl J Med. 2012;367;1998-2005. http://dx.doi.org/10.1056/NEJMoa1206809

11. Heleno B, Thomsen MF, Rodrigues DS, Jørgensen KJ, Brodersen J. Quantification of harms in cancer screening trials: literature review. BMJ. 2013;347:f5334. http://dx.doi.org/10.1136/bmj.f5334

12. Rasmussen K, JørgensenKJ, GøtzschePC. Citations of scientific results and conflicts of interest: the case of mammography screening. Evid Based Med. 2013; 8(3):83-9. PMid:23635839 PMCid:PMC3664368. http://dx.doi.org/10.1136/eb-2012-101216

13. Hospital do Câncer de Barretos. Outubro Rosa 2013 no Hospital de Câncer de Barretos [internet]. Disponível em: http://www.hcancerbarretos.com.br/institucional/unidades/82-noticias-institucional/938-uma-cidade-rosa.

14. Sociedade Brasileira de Mastologia. Como lidar com os temores sobre o câncer de mama [internet]. Disponível em: http://www.sbmastologia.com.br/index/index.php/rastreamento-e-diagnostico/61-como-lidar-com-os-temores-sobre-o-cancer-de-mama.

15. Federação Brasileira de Instituições Filantrópicas de Apoio à Saúde da Mama (FEMAMA). Documento de Posição da FEMAMA para o diagnóstico precoce do câncer de mama [Internet]. FEMAMA; 2010. Disponível em: http://www.femama.org.br/novo/arquivos/0.163891001286463380.pdf.

16. Ministério da Saúde (BR), Secretaria de Atenção à Saúde, Departamento de Atenção Básica. Rastreamento. Brasília: Ministério da Saúde; 2010. Disponível em: http://189.28.128.100/dab/docs/publicacoes/cadernos_ab/abcad29.pdf

17. Ministério da Saúde (BR). Novembro Azul conscientiza homens para prevenção do câncer de próstata [internet]. Disponível em: http://www.brasil.gov.br/saude/2012/11/novembro-azul-conscientiza-homens-para-prevencao-do-cancer-de-prostata

18. Instituto Nacional do Câncer (INCA). Rastreamento para o Câncer da Próstata: Diretrizes [internet]. Disponível em: http://www1.inca.gov.br/inca/Arquivos/publicacoes/diretriz_rastreamento_prostata.pdf.

19. United States Preventive Services Task Force (USPSTF) [internet]. Disponível em: http://www.uspreventiveservicestaskforce.org/prostatecancerscreening.htm.

20. Jamoulle M, Gusso G. Prevenção quaternária: primeiro não causar dano. In: Gusso G, Lopes JMC, organizadores. Tratado de medicina de família e comunidade e comunidade: princípios, formação e prática. Porto Alegre: Artmed; 2012. v. 1, p. 2015-211.

21. Norman AH, Tesser CD. Prevenção quaternária na atenção primária à saúde: uma necessidade do Sistema Único de Saúde. Cad Saúde Pública. 2009;25(9)2012-2020. http://dx.doi.org/10.1590/S0102-311X2009000900015 